\title{
Refuerzo interdisciplinar de las combinaciones numéricas básicas en Educación Primaria
}

\author{
Interdisciplinary reinforcement of basic numerical combinations \\ in Primary Education
}

\section{Susana Nieto-Isidro ${ }^{1}$ \\ María de los Ángeles Moro Domínguez²}

\begin{abstract}
Resumen: El objetivo del trabajo es la evaluación de un programa interdisciplinar de refuerzo de las combinaciones numéricas aditivas básicas mediante actividades realizadas en el Aula de Educación Física durante un trimestre, aplicado a un grupo de 25 escolares del primer curso de Educación Primaria, con un grupo paralelo de control. Se refuerza la modelización de las sumas elementales utilizando un esquema narrativo complejo y coherente, y su automatización utilizando las combinaciones numéricas básicas como "llave" para la realización de actividades asociadas a las rutinas de aula. Mediante análisis descriptivos e inferenciales intragrupo e intergrupo en diferentes momentos del trimestre, los resultados muestran una rápida disminución de los errores y del conteo, así como una adquisición significativa $(\alpha<.05)$ o muy significativa $(\alpha<.01)$ de las destrezas de cálculo elemental en el grupo participante, comparadas con su propio nivel inicial y con el comportamiento del grupo de control. Se concluye la eficacia del programa, destacando el uso de narraciones y juegos, el refuerzo diferencial de la automatización y del cálculo significativo, y la importancia del modelado y del uso de objetos contables.
\end{abstract}

Fecha de recepción: 20 de enero de 2020. Fecha de aceptación: 26 de junio de 2020.

1 Universidad de Salamanca (España), Instituto Universitario de Ciencias de la Educación, sni@usal.es, orcid.org/0000-0002-6751-9398

2 Centro de Educación Infantil y Primaria "C.E.I.P. Campo Charro", Salamanca (España), mamoro@ educacyl.es, orcid.org/0000-0002-4359-9530 
Palabras clave: matemáticas; combinaciones numéricas básicas; enseñanza primaria; interdisciplinariedad; cálculo.

\begin{abstract}
The objective of this work is the evaluation of an interdisciplinary program to reinforce basic additive numerical combinations through activities carried out in the Physical Education Classroom during a quarter, applied to a group of 25 schoolchildren of the first Primary Education course with a parallel control group. The modelling of elementary sums is reinforced using a complex and coherent narrative scheme, and their automation is reinforced using basic numerical combinations as a "key" for performing activities associated with classroom routines. By means of descriptive analysis and in-group and intergroup inferential analysis carried out at different times of the quarter, the results show a rapid decrease in errors and counting as well as a significant $(\alpha<05)$ or very significant $(\alpha<.01)$ acquisition of elementary calculation skills in the participating group, compared to their own initial level and with the behavior of the control group. It is concluded the effectiveness of the applied program, highlighting the use of narratives and games, the differential reinforcement of automation and significant calculation, and the importance of modeling and of the use of accounting objects.
\end{abstract}

Keywords: mathematics; basic number combinations; primary education; interdisciplinary research; calculus.

\title{
INTRODUCCIÓN
}

Esta experiencia se enmarca dentro de un proyecto interdisciplinar más amplio titulado "Integración de competencias matemáticas en el aula de Educación Física en el primer ciclo de Educación Primaria", galardonado con el XV Beca/ Premio de Magisterio "Perfecta Corselas" otorgado por Fundación Vicente y García Corselas (Universidad de Salamanca, España). Este proyecto de innovación didáctica pretende mostrar diversos ejemplos de uso de actividades realizadas en el Aula de Educación Física como refuerzo de diversos conceptos matemáticos en los primeros cursos de Educación Primaria.

En el caso presentado en este trabajo, se trata de reforzar tanto la automatización simple de las combinaciones numéricas aditivas básicas (los resultados 
de las sumas elementales desde 0+0 hasta 9+9), como la modelización de la suma (utilizando el resultado como base para una actividad física o manipulativa posterior), con escolares que comienzan el primer curso de Educación Primaria (5-6 años).

Para ello, consideramos diferentes aspectos que se han tenido en cuenta en el diseño del programa utilizado en esta experiencia: por una parte, cuáles son las habilidades de cálculo automático y la importancia de su adquisición en edades tempranas; las relaciones entre las Matemáticas y la Educación Física en Educación Primaria; la conveniencia del uso de juegos y narraciones en la enseñanza de las matemáticas y la importancia de las estrategias de modelización y el uso de objetos contables en el aprendizaje de la suma. Finalmente, se mostrarán los objetivos concretos del presente trabajo.

\section{LAS HABILIDADES DE CÁLCULO AUTOMÁTICO}

El dominio de adecuadas destrezas de cálculo automático cobra gran importancia, pues la aritmética se usa en una gran variedad de contextos (Bisanz et al., 2005). El cálculo automático, frente al cálculo mental reflexivo, a las estimaciones o al cálculo escrito, requiere de un proceso de adquisición ligado a la repetición, un número de veces que será diferente en función del alumno/a (Gregorio, 2004). Aragón et al., (2016) indican la necesidad no solamente de realizar un cálculo correcto y preciso (sin errores) sino también alcanzar un nivel óptimo de fluidez en dicho cálculo, como requisito para llevar a cabo tareas de mayor complejidad, y destacan que es esencial "el dominio de las combinaciones básicas de números pequeños" (p. 486). Este dominio de las combinaciones numéricas básicas es la base de las destrezas de cálculo algorítmico y estimado y es también necesario para la aritmética mental (Isaacs y Carroll, 1999; Ortega y Ortiz, 2006).

Por otra parte, gran número de estudios recientes relacionan las habilidades matemáticas posteriores con la competencia aritmética inicial (Geary et al., 2013; Mercader et al., 2017; Valencia, 2013). Autores como MacLellan (2012), Locuniak y Jordan (2008) o Jordan et al., (2009) indican que el conocimiento de las combinaciones básicas al comienzo de la educación primaria es el factor que más influye en la fluidez de cálculo en grados posteriores. Por todo ello, parece recomendable reforzar el entrenamiento de las sumas elementales en las primeras edades, pues servirá de base para los aprendizajes posteriores. 
Para reforzar esta adquisición, se ha utilizado un doble planteamiento. Por una parte se refuerza el cálculo significativo de la suma (en el sentido de "cálculo con significado") asociando el resultado de las combinaciones básicas al número de elementos que se han de manipular en las actividades propuestas. Y por la otra, se refuerza la automatización mediante el cálculo de sumas elementales durante las rutinas de aula y como "llave" para la realización de las actividades físicas.

En este sentido, Schutte et al. (2015) encontraron que para aumentar la ganancia en fluidez matemática era más eficaz realizar las operaciones de forma distribuida, es decir, con varias repeticiones a lo largo del día, que realizarlas de forma masiva una sola vez al día. Por ello, en esta experiencia se ha optado por realizar repeticiones frecuentes en diferentes momentos de la sesión de aula, asociadas a las rutinas propias del Aula de Educación Física.

\section{MATEMÁTICAS Y EDUCACIÓN FÍSICA}

Los trabajos sobre adquisición de competencias matemáticas en Educación Primaria están dirigidos generalmente a actividades realizadas en el Aula de Matemáticas; sin embargo, es posible extender estas actividades de carácter matemático a otros ámbitos del aprendizaje, como es el Aula de Educación Física. Este planteamiento interdisciplinar es especialmente interesante en las primeras etapas del aprendizaje matemático pues permite mostrar diferentes contextos de aplicación y fomentar la generalización de las nociones básicas. La idea del enfoque de las actividades matemáticas en diversos contextos es defendida por autores como Canals (2007) o Chamorro (2011); Alsina (2012) indica que "no todas las matemáticas se tienen que aprender necesariamente "durante la hora de matemáticas" ni "en la clase de matemáticas, sino que hay múltiples contextos de aprendizaje válidos para generar conocimiento matemático" (p. 13).

El Aula de Educación Física es un área especialmente afín para la interrelación con las matemáticas en las primeras edades escolares. Aunque la competencia matemática no aparece de forma específica en la legislación española como una competencia básica a la que se contribuye desde el Área de Educación Física, hay una gran coincidencia entre ambas en estos niveles, lo que permite una integración muy natural entre ellas. Según Rigal (2006), la actividad motriz actúa como estimuladora de la actividad mental favoreciendo las 
representaciones mentales; en el caso de los números y el cálculo, nos indica que "adición y sustracción son primero acciones motrices mucho antes de ser operaciones aritméticas" (p. 52); también Alsina (2016) resalta la importancia que tiene el desarrollo motor (entre otros) para el desarrollo matemático. Por otra parte, la presencia en el Aula de Educación Física de movimiento, competición, trabajo en equipo, manipulación de materiales y del propio cuerpo, etc., fomenta el aspecto lúdico y dinámico de las actividades matemáticas desarrolladas en este entorno, además de proporcionar un contexto real que permita situar el conocimiento matemático (Tucker, 2014; Rodríguez-Martín y Buscá, 2018).

Hay que tener en cuenta que realizar sin más una actividad física cualquiera no es la forma más adecuada de fomentar el aprendizaje matemático, sino que esta actividad física tiene que estar relacionada con el contenido que se desea reforzar. Así Mavilidi et al. (2017) constataron el efecto positivo de la actividad física en preescolares para el aprendizaje de contenidos de ciencias, mostrando que era la actividad física integrada, en la que los movimientos físicos realizados tenían relación con los contenidos, la que obtenía los mejores resultados frente al movimiento físico sin relación con el contenido o la no realización de actividad física. Más recientemente Mavilidi et al. (2018) obtuvieron un resultado similar en el caso de las habilidades numéricas, mostrando la relación entre el conocimiento de los números y las acciones físicas integradas, es decir, las que estaban relacionadas con el conteo. Por lo tanto, será necesario diseñar las actividades físicas de forma que no interfieran con el propio programa de Educación Física y que a la vez refuercen el contenido matemático que se desea fomentar.

Las propuestas previas de conexión entre las matemáticas y la actividad física en el sistema educativo de España están referidas habitualmente a la etapa de Educación Infantil (3-5 años). Por ejemplo, Gómez-Perancho (2014) muestra la relación entre algunos patrones psicomotores y la competencia matemática en Educación Infantil (3-4 años); Mateos et al. (2016) han realizado una experiencia de aprendizaje de conceptos matemáticos en edades tempranas (2-3 años) utilizando el cuerpo de los niños como contexto. En el caso de las nociones geométricas en Educación Infantil, destacamos las propuestas de Fernández-Díez y Arias (2013) y de Alsina et al. (2016).

Sin embargo, este planteamiento interdisciplinar es un enfoque menos habitual en el nivel de Educación Primaria (6-11 años). En los primeros cursos de Educación Primaria y dentro del proyecto de innovación didáctica en el que se enmarca el presente trabajo, Nieto-Isidro y Moro (2018) han presentado 
propuestas sobre la integración de las figuras geométricas elementales en el Aula de Educación Física y sobre el enriquecimiento de las nociones de medida de la capacidad y medida de la longitud (Nieto-Isidro y Moro, 2017a, 2017b). También Rodríguez-Martín y Buscá (2018), presentan una propuesta de proyectos cooperativos para desarrollar la competencia matemática desde la Educación Física en Educación Primaria.

\section{EL USO DE JUEGOS Y NARRACIONES EN EL APRENDIZAJE DE LAS MATEMÁTICAS}

En las primeras edades, los niños construyen los conocimientos a partir de sus experiencias cotidianas, y dentro de esas experiencias el juego tiene un papel fundamental. Autores como Bonilla (2014), Chamorro (2011), y Tucker (2014) recomiendan planificar las actividades de aprendizaje matemático en las primeras edades en forma de juego; y en el trabajo de Vogt et al., (2018) se constata una mayor ganancia de aprendizaje matemático en las actividades realizadas con un planteamiento basado en el juego, especialmente entre los preescolares de mayor capacidad. Asimismo, el juego proporciona oportunidades de aprendizaje matemático en los primeros años de la educación primaria (Cardón y Sgreccia, 2016; Gosling y McArdle, 2019). Algunas iniciativas de acercamiento a las matemáticas en las primeras edades mediante juegos en nuestro país se pueden encontrar en la revisión bibliográfica de González-Peralta et al., (2014) y en el trabajo de revisión sobre la investigación en didáctica de las matemáticas de Edo y Artés (2016).

Las narraciones también constituyen un componente clave a la hora de acercar las matemáticas a la realidad cotidiana del niño (Bonilla, 2014), pues las narraciones y los cuentos tienen la capacidad de aunar aspectos cognitivos y afectivos (Marín, 2007). Gil y Vicent (2009), describen la efectividad de un programa de enseñanza de las matemáticas a los 5-6 años basado en narraciones, muy superior a los programas basados en fichas de trabajo o en elementos manipulativos, destacando que "Otra de las características del niño cuando inicia su escolaridad es el gusto por las narraciones" (p. 71). Gómez-Ortega (2018) ilustra el aprendizaje de la suma y la resta en Educación Infantil mediante actividades enmarcadas en un cuento. Casey et al., (2004) muestran el uso de historias orales complejas de carácter épico como método para introducir conceptos matemáticos de forma progresiva, y conectados con otras materias. 
Este uso de las narraciones para potenciar el aprendizaje matemático se puede realizar utilizando libros, cuentos y relatos ya editados, como los propuestos por Marín (2013) y los recopilados por Fernández et al., (2014), o bien construyendo historias propias, como es el caso de la narración propuesta como hilo conductor en el presente trabajo.

\section{LA IMPORTANCIA DE LAS ESTRATEGIAS DE MODELIZACIÓN}

Antes de que los niños utilicen procedimientos de resolución más elaborados de la operación de suma, utilizan un conjunto de estrategias simples entre las que se encuentra la utilización de "objetos contables" que suponen un modelado directo de la operación de suma, así como el conteo con o sin ayuda desde el primer sumando o desde el sumando mayor (Baroody, 1994; Bermejo, 1990; Carpenter y Moser, 1984; Isaacs y Carroll, 1999; Orrantia 2006). Alsina (2016) nos indica que, al finalizar el segundo ciclo de la educación infantil (5-6 años), "Ios alumnos pueden resolver operaciones sencillas de suma y resta, siempre y cuando los contextos asociados con estas operaciones reflejen experiencias reales y puedan ser modeladas con materiales concretos" (p. 157). Boaler (2015), además de reconocer la importancia de tener disponibles en la memoria algunos hechos matemáticos básicos, indica que la mejor manera de incrementar el sentido numérico de los estudiantes es mediante el desarrollo de actividades que requieran de la manipulación de cantidades. En este sentido en la presente experiencia se ha fomentado la manipulación de diversos objetos contables (envases, tapones, tablillas, pinzas, etc.) cuya cantidad corresponde al resultado de las combinaciones numéricas básicas entrenadas previamente, lo que refuerza el modelado de las mismas (Tucker, 2014).

Por otra parte, Ramírez y De Castro (2016) indican que estas estrategias de modelización directa y conteo, son las elegidas prioritariamente por los niños cuando tienen libertad de elección, pasando progresivamente a un uso más frecuente de conocimientos formales como el algoritmo. Para cuantificar esta evolución progresiva de la adquisición de las combinaciones numéricas básicas, en este estudio se contabilizarán de forma explícita las respuestas de los niños dadas "con ayuda", generalmente con el concurso del conteo con los dedos (Bisanz et al., 2005; Crollen y Noël, 2015; Rigal, 2006). Con base en todo lo anterior, el objetivo de este trabajo es analizar la evolución de los dos grupos 
participantes (el grupo en el que se realiza la experiencia y un grupo de control) a lo largo del trimestre comparando la variabilidad intragrupo e intergrupo.

\section{MÉTODOLOGÍA}

\section{PARTICIPANTES Y CONTEXTO}

En esta experiencia y su posterior análisis participaron dos grupos preexistentes en el mismo centro educativo, el colegio de Educación Infantil y Primaria "C.E.I.P. Campo Charro" de Salamanca (España). Se trata, por tanto, de grupos no aleatorizados, más allá de la distribución realizada por el propio centro sobre los estudiantes matriculados. Uno de los grupos (que se denominará grupo 1, con 25 escolares) es en el que se ha realizado la experiencia. El otro grupo (que se denominará grupo 2, con 24 escolares) es uno paralelo, con el mismo programa del Centro, con la misma distribución temporal, método, manual y secuencia didáctica, y ha actuado como grupo de control.

La presencia de un grupo de control permitirá evaluar si la posible mejora de resultados en el grupo 1 se debe a la formación sobre las combinaciones numéricas básicas que, los escolares de este nivel reciben de forma paralela en el Aula de Matemáticas y/o a la propia maduración de los niños/as, y no solo a la experiencia que se ha llevado a cabo en el Aula de Educación Física.

\section{DESCRIPCIÓN DE LA PROPUESTA}

En la experiencia, se han aplicado acciones de dos tipos en las que se ha tratado de reforzar aspectos complementarios del aprendizaje de las combinaciones numéricas aditivas básicas o sumas elementales, y que se han denominado fase I y fase II.

\section{Fase I: Modelado de las combinaciones numéricas}

En la fase I, más intensiva, se ha desarrollado una Unidad Didáctica que se llevó a cabo al inicio del primer curso de Educación Primaria (5-6 años) durante dos semanas (6 sesiones), proponiendo actividades en las que el resultado 
de la suma ha de ser recordado e interpretado, pues determina el número de elementos que se van a utilizar en la acción siguiente. Por ejemplo, los escolares deben de transportar o utilizar tantos elementos (envases, tablillas, conos) como indique una suma elemental del tipo " $3+5$ ", “ $5+4$ ", " $9+2$ ", etc., y solamente pueden comenzar la actividad propuesta cuando la respuesta a la suma elemental es correcta.

Podemos ver que esta primera fase tiene un doble objetivo. Por una parte se trata de dotar de significado a la operación de suma fomentando la modelización, al utilizar objetos contables que deben manipular a continuación; y, por otra parte, motivar a los niños y niñas para obtener el resultado correcto de las operaciones formuladas, como requisito previo para realizar las actividades físicas.

Para hacer más atractivas las actividades presentadas en la Unidad Didáctica de la fase I y sobre todo para dotarlas de sentido y de un contexto común a todas ellas, se ha desarrollado un argumento narrativo coherente en el que se han insertado las diferentes pruebas y acciones físicas propuestas. Esta historia común a todas las sesiones actúa como elemento motivador y unificador, aprovechando la capacidad de los niños/as de estas edades de imaginar y crear situaciones fantásticas.

La historia desarrollada, ambientada en el salvaje Oeste, tiene como protagonista a un personaje (un nativo indio) que propone retos que los niños/as han de superar para ayudarle: alimentar al poblado indio, curar sus heridas, trasladar elementos al fuerte, rescatar a los habitantes, etc. Dentro de estos relatos se insertan las actividades propias del currículum del Aula de Educación Física (saltos, carreras, desplazamientos con y sin implementos, lanzamientos, etc.), enriquecidas con contenidos matemáticos.

En la figura 1 se muestran algunas de estas actividades: a la izquierda, varios niños están transportando envases (desplazamiento en grupo con implemento), que dentro de la historia representan alimentos que han de llevar hasta el poblado indio; antes han tenido que responder correctamente a una suma elemental que indica el número de envases que deben trasladar. A la derecha, una niña está curando con tablillas las heridas de una silueta humana creada con cuerdas (representación y conocimiento del propio cuerpo): el número de de tablillas colocadas en la silueta se corresponde con el resultado de una combinación numérica básica resuelta previamente. 

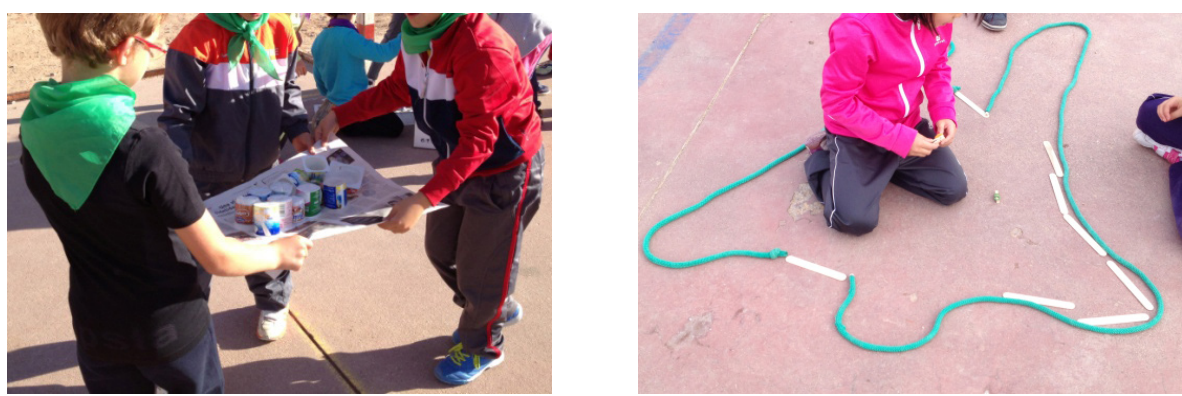

Figura 1. Ejemplos de actividades de la Unidad Didáctica en la fase I.

Además, siguiendo los planteamientos de Mavilidi et al. (2017, 2018), también se han desarrollado actividades físicas integradas, es decir, en las que la actividad física realizada está directamente relacionada con el contenido entrenado. Así, el resultado de la combinación numérica resuelta por los escolares determina el número de saltos o carreras a realizar individualmente o en grupo, o el número de elementos que intervienen en un circuito de obstáculos que han de construir y recorrer (y que dentro de la narración permite rescatar a los habitantes del poblado, sortear las trampas, etc.). Algunos ejemplos de estos circuitos se muestran en la figura 2; el número de aros (en la figura izquierda) o de conos (en la figura derecha) ha sido determinado por una combinación numérica previa:
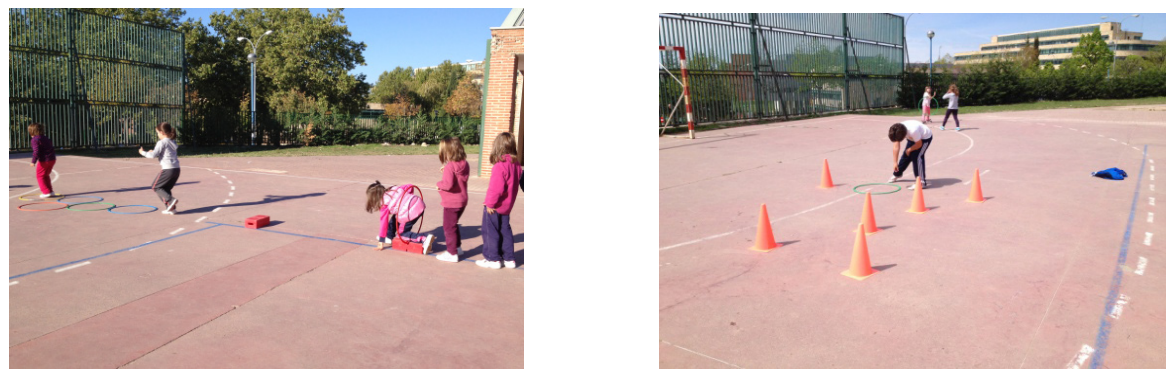

Figura 2. Ejemplo de los circuitos construidos y recorridos por los niños/as.

En cuanto al grupo de control, ha realizado las mismas actividades físicas que el grupo de la experiencia, pero en su caso no se realizan las combinaciones 
numéricas previas a las actividades ni se emplean estas como llave; es la profesora la que indica el número de saltos, carreras, elementos a transportar, etc., en cada una de las actividades propuestas.

\section{Fase II: refuerzo de la automatización}

En la fase II, más extensiva y que se ha mantenido a lo largo de todo el trimestre, se ha entrenado de forma específica la memoria de trabajo. Para ello, se han incluido las operaciones de suma dentro de las rutinas propias del Aula de Educación Física, indicando si la operación es correcta o no y repitiéndola en caso de error, pero sin actividad posterior asociada al resultado. Así, se introduce la realización diaria de sumas elementales en diferentes momentos: antes de entrar o salir del Aula de Educación Física, para recoger el material, antes de acceder a los aseos, etc. Esta rutina de "entrenamiento" y repetición frecuente pero distribuida en el tiempo (Schutte et al., 2015) fomenta la mejora de la memoria de trabajo y facilita así la automatización de las operaciones.

El grupo de control realiza las mismas rutinas propias del Aula de Educación Física, pero sin realizar la combinación numérica previa.

\section{INSTRUMENTO Y PROCEDIMIENTO DE RECOGIDA DE DATOS}

Como instrumento de recogida de datos se ha utilizado en los dos grupos una lista de control. Cada día, al finalizar la sesión, se propone a todos los miembros del grupo una combinación numérica básica, y se consignan en la lista de control el número de aciertos, el número de aciertos que se producen con ayuda (utilizando conteo con elementos auxiliares como los dedos) y las respuestas incorrectas.

La principal diferencia entre los dos grupos considerados es la frecuencia de la toma de datos: en el grupo 1 (de la experiencia) se han tomado los datos en las 30 sesiones, es decir, se ha pasado la lista de control todos los días que acudían a clase de Educación Física durante el trimestre. Sin embargo, en el grupo 2 (de control) se ha optado por espaciar la toma de datos y realizarla de forma irregular dejando pasar una quincena o incluso un mes entre cada registro. Se pretendía así que la realización de estas sumas elementales no sirviese de "entrenamiento" y distorsionase la evolución del aprendizaje en el grupo de control. 


\section{ANÁLISIS}

Para el análisis descriptivo de la evolución a lo largo del trimestre, tanto del comportamiento del grupo de la experiencia (grupo 1), como del grupo de control (grupo 2) se analizarán las representaciones gráficas de:

- La evolución de los aciertos, aciertos con ayuda y errores del grupo 1 en las dos fases de la propuesta y del grupo 2 a lo largo del trimestre.

- La diferencia (en porcentaje) de las respuestas correctas en ambos grupos para todas las sesiones en las que hay datos comparables.

También se utilizarán métodos inferenciales para estudiar las diferencias intergrupo e intragrupo en distintos momentos del trimestre. Para ello se ha optado por agrupar los datos utilizando solamente dos categorías: resultados correctos (aciertos) y resultados incorrectos (errores + conteos):

- Esta división se corresponde mejor con el objetivo de la intervención: los conteos se pueden considerar como respuestas incorrectas, pues muestran que no se ha adquirido la automatización deseada.

- Además, así se evita que en las tablas de contingencia analizadas haya casillas con ceros o con valores muy bajos, lo que podría comprometer la calidad estadística de los resultados obtenidos.

Sobre estos datos, así agrupados, se aplicará una prueba de independencia Chi-cuadrado, que es no paramétrica, adecuada en el caso del estudio de variables categóricas (Corder y Foreman, 2014). Esta prueba de independencia nos permitirá aceptar o rechazar la hipótesis de que las diferencias en el número de respuestas correctas e incorrectas sean dependientes del momento considerado (en el caso del análisis intragrupo), o del grupo considerado (en el caso del análisis intergrupo).

Se ha aplicado esta prueba Chi-cuadrado (con un nivel de significación .05 o .01) en los siguientes casos:

- Análisis intragrupo en el grupo de la experiencia, considerando las diferencias en las respuestas en tres momentos clave: al comienzo del trimestre (sesión 1), al finalizar la fase I (sesión 6) y al finalizar la fase II (sesión 30). Estos análisis nos permitirán comprobar la efectividad de la 
fase I, la efectividad de todo el programa y el efecto diferencial de la fase II respecto de la fase I.

- Análisis intergrupo entre el grupo de la experiencia y el grupo de control, considerando las diferencias en las respuestas en dos momentos clave: la situación inicial (sesión 1) y al finalizar el cuatrimestre (sesión 30).

\section{RESULTADOS}

En una primera subsección se mostrarán los resultados del análisis intragrupo del grupo 1 (de la experiencia), viendo su evolución en la fase I y la fase II y analizando si hay diferencias significativas en el comportamiento intragrupo en diferentes momentos (al comenzar la experiencia, al finalizar la fase I y al finalizar la fase II).

En una segunda subsección, se presentarán los resultados del análisis intergrupo, mostrando la relación con el grupo de control (grupo 2). Para ello se analizará la evolución del grupo 2 a lo largo del trimestre y las diferencias de comportamiento intergrupos al comienzo y al final del trimestre. Se mostrará también la evolución comparativa de las respuestas correctas de ambos grupos a lo largo del trimestre.

\section{RESULTADOS EN EL GRUPO DE LA EXPERIENCIA}

\section{Evolución del grupo 1 en la fase I}

Los niños del grupo 1, al finalizar la fase I, experimentaron una clara evolución a lo largo de estas seis sesiones (figura 3). En la sesión 1, solamente 3 niños/as del grupo fueron capaces de dar la respuesta correcta a las sumas elementales planteadas, 18 necesitaron de la ayuda de un conteo con los dedos, y 4 de ellos dieron una respuesta errónea. Sin embargo, en la sesión 6, 14 niños/as del grupo empleaban correctamente el cálculo automático sin ayuda frente a los 10 niños/as que realizaban la suma correctamente, pero requerían de ayuda exterior o de los escolares que se equivocaban en la suma (un error en esta sesión). 


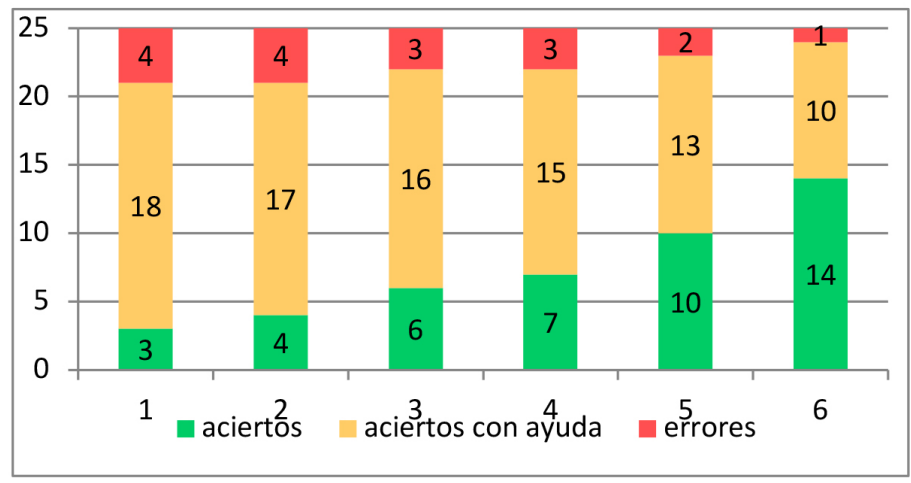

Figura 3. Evolución temporal del grupo 1 en las sesiones 1-6 (fase l).

\section{Evolución del grupo 1 en la fase II}

La figura 4 muestra la evolución positiva de las respuestas correctas del grupo 1 en la fase II, así como la disminución de las respuestas dadas con ayuda del conteo. A partir de la sesión 22 ya no se produce ningún error y, solo hay un pequeño grupo de 3-4 niños y/o niñas que mantienen el uso de la ayuda de los dedos para el conteo hasta el final de las 30 sesiones.

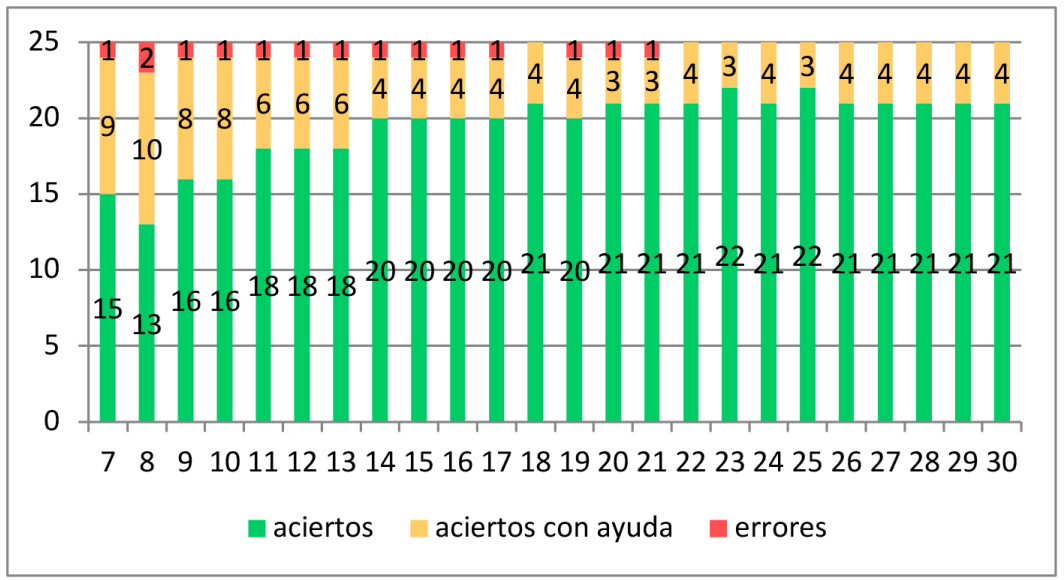

Figura 4. Evolución temporal del grupo 1 en las sesiones 7-30 (fase II). 


\section{Evolución intragrupo del grupo 1}

La evolución se ha analizado utilizando la prueba Chi-cuadrado, que nos permite estudiar si las diferencias en el número de respuestas correctas e incorrectas del grupo entre la sesión 1 y la sesión 6 (final de la fase I), la sesión 1 y la sesión 30 (final de la fase II) y las sesiones 6 y 30, son independientes del momento considerado. Los resultados obtenidos se muestran en la tabla 1:

Tabla 1. Diferencias intragrupo para el grupo 1 en las sesiones 1,6 y 30

\begin{tabular}{|c|c|c|}
\hline Chi-cuadrado de Pearson (n=50) & Valor & $\begin{array}{c}\text { Sig. asintótica } \\
\text { (bilateral) }\end{array}$ \\
\hline Grupo 1-sesión 1 vs. Grupo 1-sesión 6 & 10.784 & .001 \\
\hline Grupo 1-sesión 1 vs. Grupo 1-sesión 30 & 25.962 & .000 \\
\hline Grupo 1-sesión 6 vs. Grupo 1-sesión 30 & 4.667 & .031 \\
\hline
\end{tabular}

Se observa cómo las diferencias resultan significativas, por lo que dependen del momento considerado. Así, la situación inicial difiere de manera altamente significativa de la situación al final de la fase I $(p=.001<.01)$, y especialmente al finalizar la fase II $(p=.000<.01)$. Este resultado puede ser debido a que en la fase I, de adquisición, todavía hay un porcentaje de niños que se equivocan o recurren al conteo, mientras que en la fase II, de consolidación, disminuye el número de respuestas incorrectas (que son debidas al conteo, no a los errores) pero, sobre todo, aumenta el número de respuestas correctas dadas por los escolares.

También hay diferencia significativa entre los resultados al finalizar la fase I y al finalizar la fase II ( $p=.031<.05)$, aunque menor que las comparaciones previas. Esta diferencia significativa indica la pertinencia de ambas fases, pues corresponden a planteamientos complementarios del refuerzo de las combinaciones numéricas básicas. 


\section{RELACIÓN CON EL GRUPO DE CONTROL}

\section{Evolución del grupo 2 a lo largo del trimestre}

En la figura 5 se muestran los resultados del grupo 2 en las medidas que se han ido tomando a lo largo del primer trimestre (en este caso, espaciadas irregularmente). En este grupo no siempre han estado presentes los 24 alumnos en todas las tomas de datos (normalmente por motivos médicos), por lo que se indica también el número de escolares faltantes en cada ocasión.

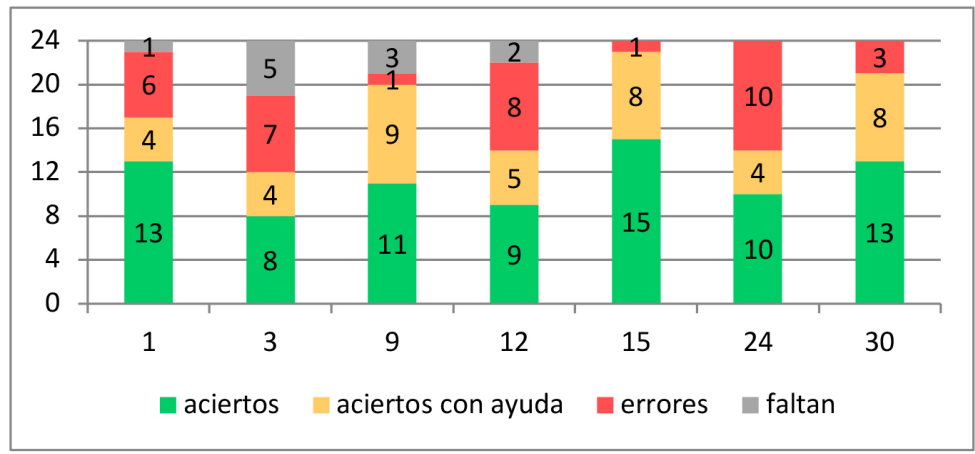

Figura 5. Distribución temporal de las respuestas del grupo 2 en las sesiones 1-30.

En la sesión 1 un total de 13 niños y niñas del grupo fueron capaces de responder correctamente a las sumas elementales planteadas, con 4 respuestas con ayuda del conteo y 6 errores en la respuesta (y 1 niño/a ausente). Esta situación inicial se mantiene sin muchas modificaciones a lo largo de todo el primer trimestre: en el grupo de control hay una gran variabilidad en los valores de los aciertos, aciertos con ayuda y errores, y no se observa ningún tipo de mejora significativa o de evolución positiva, incluso suponiendo que los datos faltantes fuesen aciertos.

\section{ANÁLISIS INTERGRUPOS}

A continuación, se realiza el análisis intergrupo utilizando de nuevo la prueba Chi-cuadrado que, nos permite estudiar en este caso si la diferencia en el número de respuestas correctas e incorrectas de los grupos 1 y 2 es independiente del 
grupo considerado. Se analizan estas diferencias en dos momentos clave, correspondientes al comienzo del trimestre (sesión 1) y una vez finalizada la experiencia al terminar el trimestre (sesión 30). Los resultados de estos análisis se muestran en la tabla 2:

Tabla 2. Diferencias intergrupo en las sesiones 1 y 30

\begin{tabular}{|c|c|c|}
\hline Chi-cuadrado de Pearson & Valor & Sig. asintótica (bilateral) \\
\hline Grupo 1-sesión 1 vs. Grupo 2-sesión $1(\mathrm{n}=48)$ & 10.137 & .001 \\
\hline Grupo1-sesión 30 vs. Grupo 2-sesión $30(\mathrm{n}=49)$ & 5.131 & .024 \\
\hline
\end{tabular}

Se puede ver que la diferencia entre los grupos en la sesión 1 es altamente significativa $(p=.001<.01)$, siendo favorable al grupo; en las figuras 3 y 5 se puede observar que la situación inicial del grupo de control era mucho mejor que la del grupo de la experiencia.

También al final del trimestre (sesión 30) hay diferencia significativa entre ambos grupos $(p=.024<.05)$, pero en este caso es favorable al grupo 1 , que termina el trimestre con una mejor distribución de respuestas correctas y sin cometer errores en las sumas, como se puede observar en las figuras 4 y 5.

\section{EVOLUCIÓN COMPARATIVA DE AMBOS GRUPOS}

Para observar con más detalle la diferencia entre las evoluciones de ambos grupos, y no solamente en los momentos puntuales del inicio (sesión 1) y final (sesión 30) del trimestre, se han emparejado los datos de las respuestas correctas dadas por los escolares correspondientes a todas aquellas sesiones en las que hay datos simultáneos del grupo 1 y del grupo 2, y que se corresponden a las sesiones 1, 3, 9, 12, 15, 24 y 30.

Dado que no en todas las ocasiones han estado presentes todos los niños/ as del grupo 2 en el aula, los datos de respuestas correctas para cada grupo se han traducido a porcentajes para realizar mejor las comparaciones. Estos emparejamientos se muestran en la tabla 3 , en la última columna se señala la diferencia de porcentajes entre el grupo 1 y el grupo 2 para cada sesión en la que hay datos comparables. 
Tabla 3. Evolución de las respuestas correctas y sus porcentajes en los grupos 1 y 2

\begin{tabular}{|c|c|c|c|}
\hline & Grupo 1 & Grupo 2 & Grupo 1-Grupo 2 \\
\hline Sesión 1 & $3(12 \%)$ & $13(56 \%)$ & $-44 \%$ \\
\hline Sesión 3 & $6(24 \%)$ & $8(42 \%)$ & $-18 \%$ \\
\hline Sesión 9 & $16(64 \%)$ & $11(52 \%)$ & $12 \%$ \\
\hline Sesión 12 & $18(72 \%)$ & $9(41 \%)$ & $31 \%$ \\
\hline Sesión 15 & $20(80 \%)$ & $15(62 \%)$ & $18 \%$ \\
\hline Sesión 24 & $21(84 \%)$ & $10(42 \%)$ & $42 \%$ \\
\hline Sesión 30 & $21(84 \%)$ & $13(54 \%)$ & $30 \%$ \\
\hline
\end{tabular}

En la última columna de la tabla 3, se observa cómo el grupo 1 tiene un comportamiento inferior al del grupo 2 en la sesión inicial (la diferencia G1-G2 es de un -44\%, a favor del grupo 2) y también en la sesión 3 (con una diferencia del $-18 \%$, también a favor del grupo 2). Sin embargo, ya en la sesión 9, una vez finalizada la fase I de la experiencia, el rendimiento del grupo 1 es mayor que el del grupo 2 (con una diferencia positiva del 12\%, a favor del grupo 1); esta diferencia se mantiene a lo largo de todas las sesiones faltantes en el trimestre.

En la figura 6, se presentan de forma gráfica los datos de la tabla 5 a lo largo del trimestre.

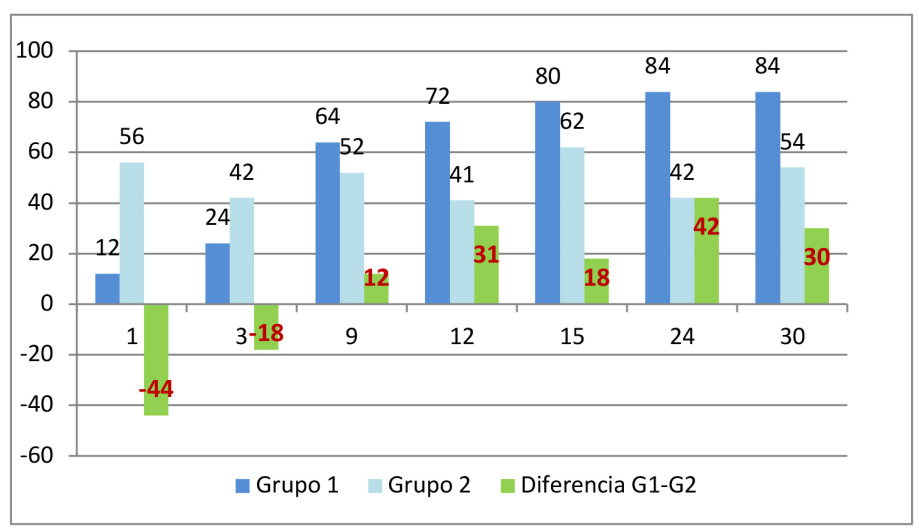

Figura 6. Porcentaje de respuestas correctas de los grupos 1 y 2 y diferencia G1-G2 
Queda patente en la figura 6 la evolución positiva de las respuestas correctas del grupo 1 a lo largo del trimestre, así como de la diferencia intergrupos (Grupo 1-Grupo 2), frente al comportamiento mucho más errático de las respuestas correctas del grupo 2.

\section{DISCUSIÓN Y CONCLUSIONES}

Se ha desarrollado y evaluado una experiencia interdisciplinar en la que las actividades realizadas en el Aula de Educación Física están dirigidas a fomentar y entrenar la adquisición de las combinaciones numéricas básicas (las sumas elementales desde el 0+0 hasta el 9+9) al comienzo del primer curso de Educación Primaria (5-6 años). Se ha utilizado un planteamiento en dos fases, que fomentan aspectos distintos del aprendizaje de la suma, y se ha cuantificado su éxito, tanto en el grupo de la experiencia como en comparación con un grupo de control.

Dentro del grupo 1, en el que se ha realizado la experiencia, se puede concluir que la propuesta ha resultado exitosa. Este grupo partía de una situación inicial en la que la mayoría de los niños/as no era capaz de realizar las combinaciones numéricas básicas, dando respuestas erróneas o teniendo que utilizar ayuda (conteo con los dedos). Esta situación ha cambiado por completo al finalizar el trimestre, mostrando unos resultados estables en los que la mayoría de los niños/as realiza las combinaciones numéricas de forma correcta y sin errores.

Este aprendizaje se ha producido en dos etapas; en la primera, denominada fase I, se ha fomentado la modelización directa de la suma y el uso de objetos contables. Ya solamente con esta fase se produce una rápida ganancia debida al aumento de respuestas correctas: con solamente 6 sesiones (dos semanas) ya superan al número de respuestas erróneas y de respuestas con ayuda.

Como factores que pueden haber contribuido al éxito de esta primera fase podemos destacar la pertinencia del uso de materiales manipulativos y objetos contables como recurso para la modelización directa de las cantidades (Baroody, 1994; Bermejo, 1990; Canals, 2007; Carpenter y Moses, 1984; Crollen y Nöel, 2015; Orrantia, 2006). También es importante la realización de actividades físicas integradas, es decir, directamente relacionadas con las cantidades calculadas en las combinaciones numéricas propuestas (Mavilidi et al., 2018), y que están insertadas en un esquema narrativo. El uso de narraciones fomenta la adquisición de contenidos matemáticos y el acercamiento de los niños a las matemáticas (Barrantes y Zapata, 2010; Bonilla, 2014; Gil y Vicent, 2009; 
Gomez-Ortega, 2018; Marín, 2007, 2013), y el uso de "sagas" o narraciones complejas permite una mejor contextualización para la resolución de problemas de contenido matemático (Casey et al., 2004).

En la fase II, en la que se utilizan estrategias de automatización ligadas a las rutinas de aula, esta ganancia se consolida de forma clara: existe una evolución positiva evidente de las respuestas correctas, así como una disminución de las respuestas dadas con ayuda del conteo. A partir de la sesión 22 ya no se produce ningún error en las operaciones básicas propuestas a los escolares, y solo hay un pequeño grupo de 3-4 niños y/o niñas que mantienen el uso de la ayuda de los dedos para el conteo hasta el final de las 30 sesiones.

Por lo tanto, la introducción de las operaciones de suma dentro de las rutinas de aula en esta fase II hace que los resultados se consoliden y que el aprendizaje resulte más estable (Gregorio, 2004); Alsina (2007) indica que "la ejecución de tareas que requieren la memoria de trabajo puede mejorarse notablemente si se entrena dicha habilidad cognitiva" (p. 330). Este sencillo gesto de plantear pequeñas sumas en diferentes momentos, como recomiendan Schutte et al. (2015), que apenas consume unos minutos en el aula, ha resultado sumamente eficaz, hasta el punto de hacer desaparecer totalmente los errores.

Cuando se realiza la cuantificación estadística de la propuesta, se observa la presencia de diferencias altamente significativas $(\alpha<.01)$ entre la situación inicial y los dos momentos explorados: al final de la fase I (cálculo significativo), y al final de la fase II (automatización). La presencia de diferencias significativas $(\alpha<.05)$ entre las dos fases muestra que ambos planteamientos deben estar presentes en el programa de refuerzo, pues inciden en aspectos complementarios de la adquisición de las combinaciones numéricas básicas.

Por otra parte, se ha comprobado que esta mejora en la adquisición de las combinaciones numéricas básicas no está relacionada con el propio avance en el conocimiento matemático o en la maduración de los niños/as durante el curso escolar. Para ello se ha analizado el comportamiento de un grupo paralelo (con las mismas características y siguiendo el mismo programa de centro) que actúa como grupo de control. Los análisis muestran que este grupo de control, aun partiendo de una situación significativamente mejor al comienzo del trimestre, no ha experimentado esta mejoría y presenta un comportamiento muy variable y poco consistente en sus respuestas a lo largo del trimestre, siendo su comportamiento significativamente inferior al finalizar la propuesta.

El caso de los aciertos con ayuda requiere de una atención especial. La contabilización de los conteos con los dedos nos proporciona una idea de cómo 
es la progresión en la adquisición de las combinaciones numéricas básicas (Bisanz et al., 2005; Carpenter y Moses, 1984; Crollen y Noel, 2015). En esta experiencia se ha detectado la presencia en ambos grupos de un subgrupo de 3-4 niños/as que al final del trimestre necesitan de la ayuda de elementos externos (mantienen el conteo con los dedos) y no adquieren la destreza de cálculo automático como sus compañeros/as. Este resultado probablemente se debe a las características psicoevolutivas particulares de estos escolares; por ejemplo, un estado madurativo anterior o un funcionamiento menos eficiente de mecanismos generales, como la memoria, o de mecanismos más específicos relacionados con el cálculo numérico (Alsina 2007; Aragón et al., 2016; Geary et al., 2013). En ese caso, estos valores de aciertos con conteo no serían fácilmente modificables por las intervenciones de carácter general como la descrita en este trabajo, sino que requerirían de un tratamiento más individualizado.

La principal limitación de este estudio es el número de escolares implicados, pues se emplean solamente dos cursos paralelos de primer curso de Educación Primaria en un único Centro escolar. Aún así, los resultados obtenidos en una muestra mayor de niños y niñas de este nivel irían seguramente en el mismo sentido que los mostrados en este trabajo, dada la alta significación encontrada en los análisis. Además, cabe destacar la fácil transferencia de esta experiencia, que puede ser replicada y adaptada de forma sencilla y con escaso coste en diferentes Centros, cursos y niveles.

Como conclusión final, se podría indicar que esta propuesta está enmarcada dentro de un proyecto más general que pretende mostrar un posible enfoque interdisciplinar para el desarrollo de algunas competencias matemáticas al comienzo de la Educación Primaria, dotándolas de un campo de aplicación dinámico y atractivo y en un entorno de juego, movimiento y actividad física integrada en el Aula de Educación Física. Como indica Rigal (2006), en la Educación Primaria "las estructuras lógico-matemáticas iniciales aparecen asociadas a esquemas sensoriomotores" (p. 303). En este sentido, la Educación Física puede actuar como generadora de contextos de aprendizaje, aplicación y enriquecimiento de los contenidos matemáticos en un entorno distinto del aula de matemáticas, como recomiendan muchos autores (Alsina, 2012; Canals, 2007; Chamorro, 2011; Nieto-Isidro y Moro, 2017a, 2017b, 2018; Rodríguez-Martín y Buscá, 2018). También el aspecto lúdico y motivador de la actividad física puede fomentar una mayor vinculación emocional de los niños/as hacia los contenidos matemáticos incluidos (Tucker, 2014, Vogt et al., 2018), mejorando su aprendizaje y adquisición en estas edades. 


\section{AGRADECIMIENTOS}

Esta experiencia forma parte del Proyecto de Innovación Didáctica "Integración de competencias matemáticas en el aula de Educación Física en el primer ciclo de Educación Primaria". Las autoras agradecen a la Fundación Vicente y García Corselas (Universidad de Salamanca, España) el apoyo a este Proyecto.

\section{REFERENCIAS}

Alsina, Á. (2007). ¿Por qué algunos niños tienen dificultades para calcular?: Una aproximación desde el estudio de la memoria humana. Revista latinoamericana de investigación en matemática educativa, 10(3), 315-333.

Alsina, Á. (2012). Hacia un enfoque globalizado de la educación matemática en las primeras edades. Números. Revista de didáctica de las matemáticas, 80, 7-24.

Alsina, Á. (2016). El currículo del número en educación infantil: un análisis desde una perspectiva internacional. PNA: Revista de Investigación en Didáctica de la Matemática, 10(3). 135-160.

Alsina, Á., Novo, M.L. y Moreno, A. (2016). Redescubriendo el entorno con ojos matemáticos: Aprendizaje realista de la geometría en Educación Infantil. Edma 0-6: Educación Matemática en la Infancia, 5(1), 1-20.

Aragón, E., Navarro, J.I. y Aguilar, M. (2016). Domain-specific predictors for fluency calculation at the beginning of primary school education. Electronic Journal of Research in Educational Psychology, 14(40), 482-499. http://doi.org/10.14204/ejrep. 40.15107

Baroody, A.J. (1994). El pensamiento numérico de los niños. Un marco evolutivo para maestros de preescolar, ciclo inicial y educación especial ( $2^{\underline{a}}$ Ed). Visor.

Bermejo, V. (1990). El niño y la aritmética. Instrucción y construcción de las primeras nociones aritméticas. Paidós Educador.

Bisanz, J., Sherman, J.L. Rasmunsen, C. y Ho, E. (2005). Development of arithmetic skills and knowledge in preschool children. In J.I.D Campbell (Ed.) The handbook of mathematical cognition, (143-162). Psychology Press.

Boaler, J. (2015). Fluency without fear: Research evidence on the best ways to learn math facts. Reflections, 40(2), 7-12.

Bonilla, F.J. (2014). El cuento y la creatividad como preparación a la resolución de problemas matemáticos. Edma 0-6: Educación Matemática en la Infancia, 3(1), 117-143.

Canals, M.A. (2007). La construcción progresiva del saber numérico desde infantil a primaria. En J.A. Fernández-Bravo, Aprender matemáticas. Metodología y modelos europeos (51-57). Ministerio de Educación y Ciencia. 
Cardón, V. y Sgreccia, N.F. (2016). Lugar que asume el juego como estrategia didáctica en clases de Matemática al inicio de la escolaridad primaria. Unión: revista iberoamericana de educación matemática, 47, 81-105.

Carpenter, T.P. y Moser, J.M. (1984). The acquisition of addition and subtraction concepts in grades one through three. Journal for Research in Mathematics Education, 15(3), 179-202. http://doi.org/10.2307/748348

Casey, B., Kersh, J E. y Young, J.M. (2004). Storytelling sagas: An effective medium for teaching early childhood mathematics. Early Childhood Research Quarterly, 19(1), 167-172. http://doi.org/10.1016/j.ecresq.2004.01.011

Chamorro, M.C. (2011). La mejora del aprendizaje del área lógico-matemática desde el análisis del currículum de Educación Infantil. Educatio Siglo XXI, 29(2), 23-40.

Corder, G.W. y Foreman, D I. (2014). Nonparametric statistics: A step-by-step approach. John Wiley \& Sons.

Crollen, V. y Noël, M.P. (2015). The role of fingers in the development of counting and arithmetic skills. Acta Psychologica, 156, 37-44. https://doi.org/10.1016/j.actpsy.2015.01.007

Edo, M. y Artés, M. (2016). Juego y aprendizaje matemático en educación infantil. Edma 0-6: Educación Matemática en la Infancia, 5(1), 33-44.

Fernández, R., Harris, C. y Aguirre, C. (2014). Propuestas para el tratamiento de la Competencia Matemática y de Ciencias a través de la literatura infantil en Educación Infantil y Primaria. Números. Revista de Didáctica de las Matemáticas, 85, 25-39.

Fernández-Díez, B. y Arias, J.R. (2013). La Expresión Corporal como fuente de aprendizaje de nociones matemáticas espaciales en Educación Infantil. Retos. Nuevas tendencias en Educación Física, Deporte y Recreación, 24, 158-164.

Geary, D. C., Hoard, M. K., Nugent, L. y Bailey, D. H. (2013). Adolescents' functional numeracy is predicted by their school entry number system knowledge. PloS one, 8(1), e54651. http://doi.org/10.1371/journal.pone.0054651

Gil, M.D. y Vicent, C. (2009). Análisis comparativo de la eficacia de un programa lúdico-narrativo para la enseñanza de las matemáticas en Educación Infantil. Psicothema, 21(1), 70-75. Gómez-Ortega, V. (2018). Una introducción a la suma y la resta en Educación Infantil a través de un cuento. Edma 0-6: Educación Matemática en la Infancia, 7(1), 82-98.

Gómez-Perancho, S. (2014). Influencia de la motricidad en la competencia matemática básica en niños de 3 y 4 años. Edma 0-6: Educación Matemática en la Infancia, 3(1), 49-73. González-Peralta, A.G., Molina, J.G. y Sánchez-Aguilar, M. (2014). La matemática nunca deja de ser un juego: investigaciones sobre los efectos del uso de juegos en la enseñanza de las matemáticas. Educación matemática, 26(3), 109-133.

Gosling, A. y McArdle, M. (2019). Mathematics through exploration and play. En S. Pope y P. Mayorga (Eds.) Enriching Mathematics in the Primary Curriculum (15-29). SAGE Publications. 
Gregorio, J.R. (2004). El cálculo en el primer ciclo de Primaria. Sigma: revista de matemáticas, 25, 71-97.

Isaacs, A.C. y Carroll, W.M. (1999). Strategies for basic-facts instruction. Teaching Children Mathematics, 5(9), 508-516.

Jordan, N.C., Kaplan, D., Ramineni, C. y Locuniak, M.N. (2009). Early math matters: kindergarten number competence and later mathematics outcomes. Developmental psychology, 45(3), 850-867. http://doi.org/10.1037/a0014939

Locuniak, M.N. y Jordan, N.C. (2008). Using kindergarten number sense to predict calculation fluency in second grade. Journal of learning disabilities, 41(5), 451-459. http:// doi.org/10.1177/0022219408321126

MacLellan, E. (2012). Number sense: The underpinning understanding for early quantitative literacy. Numeracy, 5(2), Article 3. Revista electrónica sin paginación. http://doi. org/10.5038/1936-4660.5.2.3

Marín, M. (2007). El valor matemático de un cuento. Sigma: revista de matemáticas, 31, 11-26. Marín, M. (2013). Cuentos para aprender y enseñar matemáticas en Educación Infantil (Vol. 72). Narcea Ediciones.

Mateos, A., Macías, J. y Arteaga, B. (2016). Una experiencia dentro de la conceptualización personalizada en el aula de infantil: las matemáticas y el cuerpo humano. Edma 0-6: Educación Matemática en la Infancia, 5(2), 65-79.

Mavilidi, M.F., Okely, A.D., Chandler, P. y Paas, F. (2017). Effects of integrating physical activities into a science lesson on preschool children's learning and enjoyment. Applied Cognitive Psychology, 31(3), 281-290. https://doi.org/10.1002/acp.3325

Mavilidi, M.F., Okely, A., Chandler, P., Domazet, S.L. y Paas, F. (2018). Immediate and delayed effects of integrating physical activity into preschool children's learning of numeracy skills. Journal of experimental child psychology, 166, 502-519. https://doi. org/10.1016/j.jecp. 2017.09.009

Mercader, J., Presentación, M.J. y Siegenthaler, R. (2017). Influencia de las habilidades matemáticas básicas en el rendimiento posterior. International Journal of Developmental and Educational Psychology, 3(1), 243-252. https://doi.org/10.17060/ijodaep. 2017.n1.v3.993

Nieto-Isidro, S. y Moro, M.A. (2017a). Refuerzo de las medidas de capacidad en el aula de Educación Física en los primeros cursos de Educación Primaria. Actas XVIII Congreso Internacional de Investigación Educativa: Interdisciplinaridad y Transferencia, 939-948.

Nieto-Isidro, S. y Moro, M.A. (2017b). Los lanzamientos en Educación Física: un contexto para reforzar las medidas de longitud en Educación Primaria. Actas del VIII Congreso Iberoamericano de Educación Matemática (CIBEM), 115-123. 
Nieto-Isidro, S. y Moro, M.A. (2018). Geometría en el Gimnasio: una experiencia en los primeros cursos de Educación Primaria. Edma0-6, Educación Matemática en la Infancia, 7(1), 63-81.

Orrantia, J. (2006). Dificultades en el aprendizaje de las matemáticas: una perspectiva evolutiva. Revista Psicopedagogía, 23(71), 158-180.

Ortega, T. y Ortiz, M. (2006). Jerarquía holística de las dificultades asociadas a las estrategias aditivas de cálculo mental. Enseñanza de las ciencias: revista de investigación y experiencias didácticas, 24(1), 99-110.

Rigal, R. (2006). Educación motriz y educación psicomotriz en preescolar y primaria. Acciones motrices y primeros aprendizajes. Inde.

Ramírez, M. y De Castro, C. (2016). Formalización progresiva en matemáticas: el caso de la adición en primer curso de primaria. Números, Revista de Didáctica de las Matemáticas, 93, 75-92.

Rodríguez-Martín, B y Buscá, F. (2018). Desarrollar la competencia matemática desde la Educación Física. Tándem. Didáctica de la Educación Física, 61, 66-72.

Schutte, G.M., Duhon, G.J., Solomon, B.G., Poncy, B.C., Moore, K. y Story, B. (2015). A comparative analysis of massed vs. distributed practice on basic math fact fluency growth rates. Journal of School Psychology, 53(2), 149-159. http://doi.org/10.1016/j.jsp. 2014.12.003

Valencia, E. (2013). Desarrollo del cálculo mental a partir de entrenamiento en combinaciones numéricas y estrategias de cálculo. Números. Revista de Didáctica de las Matemáticas, 84, 5-23.

Vogt, F., Hauser, B., Stebler, R., Rechsteiner, K. y Urech, C. (2018). Learning through playpedagogy and learning outcomes in early childhood mathematics. European Early Childhood Education Research Journal, 26(4), 589-603. https://doi.org/10.1080/1350 293X.2018.1487160

Tucker, K. (2014). Mathematics through play in the early years ( $3^{\underline{a}}$ Ed.) SAGE Publications.

Susana Nieto-Isidro

Dirección: Departamento de Matemática Aplicada, Facultad de Ciencias

Universidad de Salamanca

$\mathrm{C} /$ del Parque, 2

37008 Salamanca (España)

Teléfono: $\quad$ +34 923294400 ext 1579 